\title{
Anxiety and Stress in In-Service Chinese University Teachers of Arts
}

\author{
Meihua $\operatorname{Liu}^{1} \&$ Yi Yan ${ }^{2}$ \\ ${ }^{1}$ Department of Foreign Languages and Literatures, Tsinghua University, Beijing, China \\ ${ }^{2}$ Language Center, Tsinghua University, Beijing, China \\ Correspondence: Meihua Liu, Department of Foreign Languages and Literatures, Tsinghua University, Beijing, \\ China. E-mail: liumeihua@mail.tsinghua.edu.cn
}

Received: November 9, 2019

Accepted: January 12, 2020

Online Published: January 16, 2020

doi:10.5430/ijhe.v9n1p237

URL: https://doi.org/10.5430/ijhe.v9n1p237

\begin{abstract}
As revealed by literature, anxiety and stress are complicated yet serious issues among teachers at all educational levels. Though widely studied, research on them often focuses on pre-service or primary and middle school teachers, with little research on in-service university teachers. It is especially so in China. The present study thus examined anxiety, stress and their relations with demographic variables in in-service university teachers in China. 256 teachers from various universities in China answered the Demographic Questionnaire, the Teaching Anxiety Scale and the Teacher Stress Inventory. Analyses of the data revealed the following main findings: (1) the participants were under great stress, but they were generally not so anxious about teaching, (2) teaching anxiety was generally significantly negatively correlated with age, professional title and years of teaching, while teacher stress was significantly negatively related to professional title, and (3) overall teacher stress, professional title and age were powerful predictors for teaching anxiety, while years of teaching, overall teaching anxiety and its subscales were powerful predictors for teacher stress. Based on these findings, some implications are discussed.
\end{abstract}

Keywords: anxiety, stress, demographic variables, relation, in-service university teachers

\section{Introduction}

The assessment of occupational anxiety and stress in teachers has been an increasingly important consideration in continuing their profession (Fimian, 1984). Researchers have been using measures such as the Anxiety Test Scale (Mandler \& Sarason, 1952), the State-Trait Anxiety Inventory (Spielberger, 1966), and the Teaching Anxiety Scale (Parsons, 1973) to measure teaching anxiety, and the Teacher Stress Inventory (Fimian, 1984) to measure teacher stress. Numerous studies have revealed that teaching can be stressful and many teachers at different school levels suffer from anxiety and stress to varying degrees (Ahmed \& Julius, 2015; Ameen, Guffey, \& Jackson, 2002; Boyle, Borg, Falzon \& Baglioni, 1995; Desouky \& Allam, 2017; Fimian\& Blanton, 1987; Kyriacou, 2001; Olthuis, Watt \& Stewart, 2014; Pithers\& Fogarty, 1995; Robinson \& Clay, 2005). These studies also show that there are considerable individual differences in proneness to anxiety and stress, which can be affected by various factors such as personality dispositions, gender, education, age, teaching experiences and school characteristics (Betoret, 2006; Kyriacou, 2001; Peker\&Halat, 2009; Wang \& Wang, 2015). In addition, anxiety and stress can lead to serious consequences such as poor teaching effectiveness, low self-esteem, and poor teacher-student relationship (Betoret, 2006; Keavney \& Sinclair, 1978; Kyriacou, 2001).

Clearly, teaching anxiety and teacher stress are complex issues, thus deserving further research. In addition, though some studies reveal that anxiety and stress are related to teachers' demographic variables (Peker \& Halat, 2009; Wang \& Wang, 2015; Yazici \& Altun, 2013), more research is needed to reveal a fuller picture. Moreover, most empirical studies focus on pre-service or primary and middle school teachers, with little research targeting university teachers, as reviewed below. This is especially so in Chinese contexts where gather a huge group of teachers but far from enough research has been done to explore their anxiety and stress (Wang \& Wang, 2015). Hence, the present study aimed to explore in-service Chinese university teachers' anxiety and stress as well as their relations with demographic variables. 


\section{Literature Review}

\subsection{Teaching Anxiety}

Anxiety is a subjective feeling of tension, apprehension, nervousness and worry (Spielberger, 1983). As a transitory state (Spielberger, 1966), teaching anxiety is the anxiety experienced in relation to teaching activities that involve the preparation and execution of classroom activities (Gardner \& Leak, 1994). To measure teaching anxiety, Parsons (1973) designed the 29-item Teaching Anxiety Scale (TAS), which then has been subjected to various validation checks and proves to be highly reliable and consistent (Keavney \& Sinclair, 1978; Parsons, 1973). Research using the TAS as well as other instruments like the State-Trait Anxiety Inventory (Spielberger, 1983) has been done on teachers, especially pre-service elementary and post-secondary teachers, of various school subjects, such as mathematics (Levine, 1996; Peker, 2009; Peker \& Halat, 2009), law (Joseph, 1975), and foreign languages (Agustiana, 2014; Hismanoglu, 2013; Machida, 2011). These studies, as well as others targeting teachers of no specific school subjects (Ahmed \& Julius, 2015; Ameen et al., 2002; Cubukcu, 2013; Gardner \& Leak, 1994; Olthuis et al., 2014; Robinson \& Clay, 2005; Westerback, 1982; Yazici \& Altun, 2013), show that teaching anxiety in general is a problem for a significant number of educators, pre-service and student teachers in particular. Most of the anxieties they experience center around such problems as evaluation of teaching, maintaining discipline, students' perceptions of teachers, knowledge of subject matter, correcting mistakes, and shortage of materials. These studies also reveal that differences exist in teaching anxiety between male and female teachers, and between those with different education levels, professional titles, ages and years of teaching (Morton, Vesco, Williams \& Awender, 1997; Peker \& Halat, 2009; Wang \& Wang, 2015).

For example, Machida (2011) recruited 133 Japanese elementary school teachers of English and found that 77.4\% of them were anxious about their own English proficiency and 90.2\% of them were anxious about teaching English due to lack of experience, training for teaching English and lack of confidence in English communication. The study also revealed that support by the city board of education was important for diminishing teachers' anxiety. Yazici and Altun (2013) investigated anxiety levels in 286 teacher candidates who were in their senior year in college. They found that concerns regarding finding a job did not have a significant effect on the anxiety scores and that there was no significant differences in anxiety scores between female and male teacher candidates. Nevertheless, Morton et al.'s (1997) research of approximately 1000 student teachers in Canada found that females showed higher anxiety ratings than males but were comparable to males after practice teaching and that females in the lower grades division showed higher anxiety scores. The study concluded that student-teacher anxieties were related to demographic, experiential and dispositional variables. These findings were supported by subsequent research (Wang \& Wang, 2015; Zhang \& Niu, 2008). Four hundred middle school teachers aged above 20 in China participated in Zhang and Niu's (2008) study, which showed that the teachers were almost all anxious or depressed to varying degrees, with nearly half being highly anxious and depressed. The study also revealed differences in anxiety and depression levels between gender, disciplines, years of teaching experience, and location of schools. Wang and Wang (2015) studied anxiety in 72 Chinese college English teachers, which revealed that $38 \%$ to $55 \%$ of the participants felt anxious because of such reasons as having little time and energy to look after families, spending too much time in preparing for lessons and thus worrying about research, promotion, being lack of relevant training, not having adequate resources for teaching, and having difficulty organizing and using reasonably different kinds of teaching resources. And the participants were different in state anxiety in regard to gender, professional titles, educational levels, years of teaching. Even so, the teachers surveyed were generally confident in their teaching outcomes and were satisfied with the gains for their hard work.

Meanwhile, researchers have found that the anxiety experienced by pre- and in-service teachers have negative impacts on them and their teaching effectiveness (Joseph, 1975; Silvernail \& Costello, 1983). For example, Keavney and Sinclair (1978) found that high teaching anxiety could lead to withdrawing from the teaching profession, student anxiety, low acceptance of self and others, low job satisfaction, reduced student motivation, diminished teaching effectiveness, low pupil/teacher rapport, and low student achievement. In addition, various sources have been identified to result in teaching anxiety such as value conflicts, student behavior, workload, faculty relations, academic demands, and knowledge of subject matter (Coates \& Thoresen, 1976; Joseph, 1975; Merç, 2011). For example, Coates and Thoresen (1976) identified five main sources of classroom teacher anxiety: time demands, difficulties with pupils, large class enrollments, financial constraints, and lack of educational resources. Merç's (2011) qualitative study revealed six main categories as the sources of foreign language student teacher anxiety: students and class profiles, classroom management, teaching procedures, being observed, mentors, and miscellaneous extra work. İpek's (2016) qualitative study of 32 non-native EFL (English as a foreign language) teachers revealed that making mistakes, fear of failure, and using the native language were the major sources of 
anxiety in those teachers.

\subsection{Teacher Stress}

Similar to anxiety, stress is also an influential factor in a profession, which refers to non-specific responses of living organisms to noxious stimuli (Selye, 1976). Teacher stress refers to "the experience by a teacher of unpleasant, negative emotions, such as anger, anxiety, tension, frustration or depression, resulting from some aspect of their work as a teacher" (Kyriacou, 2001, p.28).

Teacher stress has received increasing attention over recent decades (Boyle et al., 1995; Desouky \& Allam, 2017; Ferguson, Frost \& Hall, 2012; Fimian \& Blanton, 1987; Kyriacou, 2001; Pithers \& Fogarty, 1995). To measure teacher stress, the Teacher Stress Inventory (TSI) was developed and has been tested across different teacher groups and teaching situations and proved to be highly reliable and valid (Fimian, 1984, Fimian \& Blanton, 1987; Fimian \& Fastenau, 1990). Fimian's (1984) analyses of various samples showed that the 42-item TSI had six subscales: personal/professional stressors; professional distress; discipline and motivation; emotional manifestations; biobehavioural manifestations; and physiological-fatigue manifestations. Fimian and Blanton's (1987) study of 413 pre-professional trainees and first-year special education teachers indicated that the 38-item TSI had four factors: classroom stress, professional distress, emotional-fatigue manifestations, and biobehavioural manifestations. Boyle et al.'s (1995) study of 710 full-time primary school teachers revealed that the 20-item TSI had five dimensions: pupil misbehavior, time resource difficulties, professional recognition needs, and poor relationships, and workload. The study also revealed that workload and student misbehavior accounted for most of the variance in predicting teaching stress.

Kyriacou (2001) believed that sources of teacher stress included maintaining discipline, time pressures and workload, coping with change, being evaluated by others, dealing with colleagues, self-esteem and status, administration and management, role conflict and ambiguity, and poor working conditions. Hiebertt and Farber's (1985) review of 71 articles on teacher stress revealed that common sources of teacher stress were interpersonal interactions, working conditions, time pressures, role ambiguity, and inadequate preparation. The review also showed that some individual teachers suffered from stress-induced tension headaches and experienced real pain. Betoret's (2006) study of 247 Spanish secondary school teachers uncovered that teachers with a high level of self-efficacy and more coping resources reported suffering less stress and burnout than teachers with a low level of self-efficacy and fewer coping resources, and vice versa.

As reviewed, teaching anxiety and teacher stress are big and complex issues, which can be attributed to various sources and result in serious consequences. In addition, though some studies show that they are related to demographic variables (Peker \& Halat, 2009; Wang \& Wang, 2015; Yazici \& Altun, 2013), further research is needed to explore anxiety and stress in teachers at different educational levels or institutional settings considering the large population of teachers and the huge diversity of the population. As discussed in Wang and Wang (2015), not much empirical research has been done on teacher anxiety and stress in Chinese contexts. Moreover, as reviewed, most studies on teaching anxiety and teacher stress focused on pre-service or primary and middle school teachers, with little research targeting university teachers. For these reasons, the present study aimed to explore anxiety, stress and their relations with demographic variables in in-service Chinese university teachers and sought to answer the following research questions:

(1) What are the profiles of anxiety and stress in Chinese university teachers of arts?

(2) How do teaching anxiety and teacher stress relate to demographic variables?

(3) What factors predict teaching anxiety and teacher stress?

\section{Methods}

Participants. A total of 256 (66 male and 190 female) teachers from different universities in China participated in the present study. With an average of $17.53(\mathrm{SD}=9.99)$ years of teaching, the participants' teaching experiences ranged from 1 to 41 years. Among them, 112 (43.75\%)aged40 to 49, 97 (37.89\%) were in their thirties, $36(14.06 \%)$ were in their fifties and $11(4.3 \%)$ in their twenties; $124(48.44 \%)$ were masters, 109 (42.58\%) were Ph.Ds, and $23(8.98 \%)$ bachelors; $130(50.78 \%)$ were lecturers or assistant professors, $88(34.38 \%)$ were associate professors, $31(12.11 \%)$ professors and $7(2.73 \%)$ assistant lecturers.

Instruments. The participants answered an 8-item demographic questionnaire, the 33-item Teaching Anxiety Scale, the 18-item Teacher Stress Inventory, and an open-ended question "As a university teacher, my anxiety mainly comes from ". 
The Demographic Questionnaire. This questionnaire aimed to collect demographic data about the participants like gender, age, education, major, university of work, professional title, years of teaching, and language of instruction.

The Teaching Anxiety Scale. With a reliability score of .900 in the present study, the 32-item Teaching Anxiety Scale (TAS) was adapted from that developed by Parsons (1973). The original 29-item TAS intended to measure anxiety levels in K1-12 school teachers. To better fit the present situation, several modifications were made: 1) items about parent observation/conference were deleted, 2) items about promotion and research were added, and 3) words "pre-service" and "other interns/teachers on the program" were changed to be "in-service" and "colleagues/other teachers" respectively. Consequently, the resultant TAS used in the present study had 33 items, all of which were on a 5-point Likert scale, ranging from 'Never', 'Infrequently', 'Occasionally', 'Frequently', to 'Always' with values of 1 to 5 assigned to each of the descriptors respectively.

The Teacher Stress Inventory. With a reliability score of .897 in the present study, the 18-item Teacher Stress Inventory (TSI) was modified from the original 20-item TSI designed by Boyle et al. (1995), aiming to measure the level of stress in school teachers. To better fit the present situations, the following modifications were made: 1) items about rest period and pupil behavior and attitudes were deleted, 2) items about research, personal development, workload, the use of technology in education, and colleague relationship were added, and 3) words "pressure from parents" were changed to be "pressure from family", "pupil" was changed to be "student", and "pressure from headteacher and education officers" were changed to be "pressure from the Department and the University". Thus, the final TAS used in the present study had 18 items, all of which were on a 5-point Likert scale, ranging from 'No Stress', 'Mild Stress', 'Moderate Stress', 'Much Stress' to 'Extreme Stress' with values of 0 to 4 assigned to each of the descriptors respectively.

Procedure and data analysis. The questionnaires were translated into Chinese and double-checked by two researchers proficient in both Chinese and English, which were then made available online to teachers of all subjects in universities in China. Surprisingly, the collected questionnaires in two weeks were all answered by teachers of arts. The Teaching Anxiety Scale and the Teacher Stress Inventory were subjected to rotated (varimax) principal components analysis to reveal their underlying components. Then correlation analyses were conducted to explore the correlations within and between TAS and TSI scales, and between TAS and TSI scale and demographic variables. To identify the profiles of teaching anxiety and teacher stress of the sample, means and standard deviations of TAS and TSI scales were computed. Finally, multiple stepwise regression analyses were run to explore predictors of teaching anxiety and teacher stress. The responses to the open-ended question were analyzed in terms of themes (e.g., inadequate salary and poor administration), the results of which were incorporated into those of survey data.

\section{Results}

Both the Teaching Anxiety Scale (TAS) and the Teacher Stress Inventory (TSI) were subjected to rotated (varimax) principal components analysis before any statistical analysis was conducted, because some modifications were made to the original scales in the study. The analysis of the TAS yielded six factors, with eigenvalues ranging from 1.07 to 9.175. Accounting for $29.596 \%$ of the total variance, Factor 1 (TAS1), called Concern about Classroom Teaching, had 16 items reflective of concerns about classroom teaching such as lesson preparation, responding to students' questions, teacher-student relationship, observing classroom teaching, and presenting teaching. The second factor (TAS2), called Interest in Teaching, had 5 items indicative of interest and confidence in teaching as a profession and explained $10.344 \%$ of the total variance. The third factor (TAS3), called Concern about Research, had 4 items and accounted for $6.19 \%$ of the total variance. Factor 4 (TAS4), called Time Difficulty, had 4 items suggesting difficulty in planning time for different work, which accounted for $5.468 \%$ of the total variance. Factor 5 (TAS5) and Factor 6 (TAS6) both had 2 items and explained $4.864 \%$ and $3.451 \%$ of the total variance respectively. TAS5 items were concerned with the effects of teaching, while TAS6 items expressed concerns about promotion.

The analysis of the TSI produced four factors, with eigenvalues ranging from 1.024 to 6.866 , slightly different from the five factors yielded in Boyle et al. (1995). The first factor (TSI1), called Time Resource Difficulties, had 7 items expressing stress related to lack of sufficient time for individual instruction, a vaguely detailed syllabus, shortage of equipment and poor facilities, large class size, administrative work, and personal development. Factor 2 (TSI2), called Workload, included 4 items suggesting too much work, responsibility for students, difficult courses, research, and heavy workload. The third factor (TSI3), called Colleague Relations, had 4 items involving pressure from the Department and the University, family, and colleagues. Factor 4 (TSI4), called Professional Recognition Needs, involved 3 items related to career structure, salary, and recognition for good teaching. These four factors were named with reference to Boyle et al. (1995) and accounted for 38.147\%, 9.728\%, 7.299\% and 4.409\% of the total variance, respectively. 


\subsection{Correlations within and between TAS and TSI Scales}

Correlational analyses were run to examine the relations within and between the TAS and TSI scales (To avoid Type I errors, Bonferroni correction was carried out in the analyses, with the threshold of p lowered from .05 to be at .004 and from .01 to be at .0008.). The results are reported in Table 1 .

Table 1. Correlations within and between TAS and TSI scales $(\mathrm{N}=256)$

\begin{tabular}{|c|c|c|c|c|c|c|c|c|c|c|c|}
\hline & TAS2 & TAS3 & TAS4 & TAS5 & TAS6 & TAS & TSI1 & TSI2 & TSI3 & TSI4 & TSI \\
\hline TAS1 & $.626^{* * *}$ & $.281 * *$ & .062 & $.647 * *$ & $.323 * *$ & $.894 * *$ & $.374 * *$ & $.349 * *$ & $.320 * *$ & $.283 * *$ & $.384 * *$ \\
\hline TAS2 & 1 & $.300 * *$ & .071 & $.495 * *$ & $.375 * *$ & $.763 * *$ & $.266 * *$ & $.305^{* *}$ & $.321 * *$ & $.380 * *$ & $.351 * *$ \\
\hline TAS3 & & 1 & $.411 * *$ & .152 & $.534 * *$ & $.582 * *$ & $.366^{* *}$ & $.451 * *$ & $.361 * *$ & $.506^{* *}$ & $.465 * *$ \\
\hline TAS4 & & & 1 & -.051 & $.309 * *$ & $.318 * *$ & $.469 * *$ & $.469 * *$ & $.414 * *$ & $.502 * *$ & $.525^{* *}$ \\
\hline TAS5 & & & & 1 & $.315^{* *}$ & $.652 * *$ & $.225 * *$ & $.192 * *$ & $.227 * *$ & $.180^{*}$ & $.238 * *$ \\
\hline TAS6 & & & & & 1 & $.591 * *$ & $.290 * *$ & $.372 * *$ & $.412 * *$ & $.550 * *$ & $.439 * *$ \\
\hline TAS & & & & & & 1 & $.481 * *$ & $.501 * *$ & $.470 * *$ & $.519 * *$ & $.556^{* *}$ \\
\hline TSI1 & & & & & & & 1 & $.732 * *$ & $.737 * *$ & $.634 * *$ & $.920 * *$ \\
\hline TSI2 & & & & & & & & 1 & $.649 * *$ & $.740 * *$ & $.876^{* *}$ \\
\hline TSI3 & & & & & & & & & 1 & $.658 * *$ & $.867 * *$ \\
\hline TSI4 & & & & & & & & & & 1 & $.832 * *$ \\
\hline TSI & & & & & & & & & & & 1 \\
\hline
\end{tabular}

TAS1 = Concerns about Classroom Teaching; TAS2 = Interest/Confidence in Teaching

TAS3 = Concerns about Research; TAS4 = Time Difficulty

TAS5 $=$ Concerns about Effects of Teaching; TAS6 = Concerns about Promotion

TAS $=$ Teaching Anxiety Scale

TSI1 $=$ Time Resource Difficulties; TSI2 $=$ Workload

TSI3 = Colleague Relation; TSI4 $=$ Professional/Recognition Needs

TSI $=$ Teacher Stress Inventory

coefficient of determination: small $=r \leq 0.1 ; \quad$ medium $=r=0.3$; large $=r \geq 0.5$ (Cohen, 1988)

As seen from Table 1, all TAS scales were significantly positively related to each other $(r=.281 \sim .647, \mathrm{p} \leq .0008)$ and the overall TAS $(r=.318 \sim .894, \mathrm{p} \leq .0008)$ except for the correlation between TAS5 and TAS3 and between TAS5 5 and TAS4, so were the TSI scales to each other $(r=.634 \sim .740, \mathrm{p} \leq .0008)$ and the overall TSI $(r=.832$ $\sim .920, \mathrm{p} \leq .0008$ ), with a large effect size for all the coefficients. Table 1 also shows that the TAS scales were highly positively related to TSI scales $(r=.180 \sim .556, \mathrm{p} \leq .004)$, with a medium to large effect size for all the coefficients, meaning that a more anxious teacher tended to be under greater stress.

\subsection{Correlations between TAS and TSI Scales and Demographic Variables}

Correlational analyses were run to examine the relations between the TAS and TSI scales and the participants' demographic variables (see Table 2) (To avoid Type I errors, Bonferroni correction was carried out in the analyses, with the threshold of $\mathrm{p}$ lowered from .05 to be at .004 and from .01 to be at .0008.). 
Table 2. Correlations between TAS and TSI scales and demographic variables $(\mathrm{N}=256)$

\begin{tabular}{lccccc}
\hline & Gender & Age & Education & Title & Years of teaching \\
\hline TAS1 & -.050 & $-.252^{* *}$ & -.111 & $-.240^{* *}$ & -.146 \\
TAS2 & -.125 & $-.207^{*}$ & .032 & $-.184^{*}$ & $-.188^{*}$ \\
TAS3 & $.265^{* *}$ & -.030 & $-.300^{* *}$ & $-.283^{* *}$ & .058 \\
TAS4 & .056 & .004 & -.139 & -.110 & -.071 \\
TAS5 & -.079 & -.151 & .038 & -.093 & -.104 \\
TAS6 & .090 & $-.247^{* *}$ & -.078 & $-.442^{* *}$ & $-.211^{*}$ \\
TAS & .009 & $-.246^{* *}$ & -.145 & $-.323^{* *}$ & -.164 \\
TSI1 & -.022 & .032 & -.139 & -.042 & .053 \\
TSI2 & .011 & .051 & -.088 & -.058 & .079 \\
TSI3 & -.106 & -.053 & -.030 & -.108 & -.035 \\
TSI4 & .009 & -.055 & -.098 & $-.245^{* *}$ & -.068 \\
TSI & -.032 & .000 & -.108 & -.114 & .017 \\
\hline
\end{tabular}

Notes: $* \mathrm{p} \leq .004 ; * *=\mathrm{p} \leq .0008$

coefficient of determination: small $=r \leq 0.1 ; \quad$ medium $=r=0.3$; large $=r \geq 0.5$ (Cohen, 1988)

As reported in Table 2, TAS1was significantly inversely related to age $(r=-.252, \mathrm{p} \leq .0008)$ and professional title $(r$ $=-.240, \mathrm{p} \leq .0008)$, TAS2 was significantly negatively correlated with age $(r=-.207, \mathrm{p} \leq .004)$, professional title $(r=$ $-.184, \mathrm{p} \leq .004)$ and years of teaching $(r=-.188, \mathrm{p} \leq .004)$, TAS3 was significantly positively related to gender $(r=$ $-.265, \mathrm{p} \leq .0008)$ but negatively to education level $(r=-.300, \mathrm{p} \leq .0008)$ and professional title $(r=-.283, \mathrm{p} \leq .0008)$, TAS6 was significantly inversely related to age $(r=-.247, \mathrm{p} \leq .0008)$, professional title $(r=-.442, \mathrm{p} \leq .0008)$ and years of teaching $(r=-.211, \mathrm{p} \leq .004)$, and TAS was significantly inversely related to age $(r=-.246, \mathrm{p} \leq .0008)$ and professional title $(r=-.323, \mathrm{p} \leq .0008)$. These findings indicated that the older a teacher was, the less concerned he/she was about classroom teaching and promotion yet the more interested and confident in teaching, that a teacher who held a higher professional title tended to be less concerned about classroom teaching, research and promotion, and more interested/confident in teaching, that the longer a teacher was in the profession, the more interested/confident in teaching and less concerned about promotion he/she was, and that male participants and teachers with higher education levels tended to be less concerned with research. Moreover, older teachers and those with higher professional titles tended to be less anxious in general.

Meanwhile, of the TSI scales, only TSI4 was significantly negatively correlated with professional title $(r=-.245, \mathrm{p}$ $\leq$.0008). This meant that teachers with higher professional titles tended to feel less stressed for professional/recognition needs (such as promotion, salary and recognition of good teaching).

\subsection{Teaching Anxiety and Stress Levels}

To explore the participants' anxiety and stress levels, means and standard deviations of TAS and TSI scales were computed, the results were presented in Table 3 . 
Table 3. Means and Standard Deviations (SDs) of TAS and TSI Scales $(\mathrm{N}=256)$

\begin{tabular}{ccccc}
\hline & Mean & SD & Skewness & Kurtosis \\
\hline TAS1 & 2.15 & .61 & .29 & -.26 \\
TAS2 & 2.04 & .79 & .64 & -.24 \\
TAS3 & 3.63 & .87 & -.34 & -.38 \\
TAS4 & 3.62 & .82 & -.28 & -.28 \\
TAS5 & 2.31 & .85 & .22 & -.54 \\
TAS6 & 3.35 & 1.12 & -.46 & -.53 \\
TAS & 2.54 & .53 & .13 & -.35 \\
TSI1 & 2.97 & .74 & -.15 & -.09 \\
TSI2 & 3.34 & .77 & -.34 & .098 \\
TSI3 & 2.95 & .83 & -.29 & .009 \\
TSI4 & 3.41 & .93 & -.45 & -.21 \\
TSI & 3.12 & .70 & -.47 & .297 \\
\hline
\end{tabular}

As previously described, both TAS and TSI were 5-point Likert scales, with values of 1-5 and 0-4 assigned to each of the item descriptors, respectively. Thus, a score of more than 4, 3-4 and below 3 on a TAS scale meant high, medium and low anxiety respectively, and a score of more than 3,2-3 and below 2 on a TSI scale meant high, medium and low stress respectively.

As shown in Table 3, the participants scored below 3, the scale midpoint, on TAS1, TAS2, TAS5 and TAS (mean $=.204 \sim .254)$ but more than 3 on the other TAS scales (mean = 3.35 3.63). These findings suggested that the participants were generally not worried about classroom teaching or the effects of their teaching and that they were generally interested and confident in teaching and being good teachers. They also showed that the participants were generally anxious about research, allocating time for different work (like balancing time for research and teaching, administrative work, and too much work unrelated to teaching and research), and promotion. These findings were largely consistent with the participants' responses to the open-ended question. Analyses of the 212 responses collected revealed 13main sources of anxiety: research (92/43.4\%), promotion (63/29.7\%), administration $(33 / 15.6 \%)$, inadequate salary $(26 / 12.3 \%)$, teacher evaluation $(19 / 8.96 \%)$, heavy workload $(17 / 8.02 \%)$,personal development (15/7.08\%), balancing time and work between research, teaching and family (especially looking after children) (14/6.6\%), heavy teaching load (8/3.77\%), teacher-student relation (6/2.83\%), big class $(1 / 0.47 \%)$, colleague relation $(1 / 0.47 \%)$ and health $(1 / 0.47 \%)$. Almost half of $(43.4 \%)$ the respondents were concerned with research in that it was (rather) difficult to successfully apply for nation-wide research projects and funds, to do good research, and publish papers, especially in top journals such as those indexed in CSSCI, SCI, SSCI and A\&HCI, while universities set high demands on research. The second great source of anxiety was the promotion system (29.7\%) in that it was often unfair and that the participants had to face the risk of losing jobs if they were not promoted to be associate professors. Thirty-three respondents were not satisfied with the administration in their universities, mainly due to poor management, unreasonable interference and checks, low efficiency, numerous meaningless meetings, unfair treatment, narrow-minded leaders, and frequent reforms. Inadequate salary and the heavy financial burden it thus caused, especially in big cities was reported by $26(12.3 \%)$ respondents to be a source of their anxiety. The teacher evaluation system was anxiety-provoking because it was often unfair, unreasonable and not objective. Seventeen $(8.02 \%)$ respondents reported feeling anxious about heavy workload in that they had to handle various kinds of work including those unrelated to research and teaching and thus had little time for personal life and families. Meanwhile, 15(7.08\%) participants were anxious about personal development because they wanted to catch up with the latest development in their fields and to be updated with newest teaching methodologies but they lacked opportunities and/or support for training and/or further education.

In addition, as seen from Table 3, the respondents scored 3.34 on TSI2, 3.41 on TSI4 and 3.12 on TSI, far exceeding the scale midpoint 2.5 , meaning that they were under great stress of workload and professional/recognition needs and that their overall stress level was quite high. In addition, they scored 2.97 on TSI1 and 2.95 on TSI3, indicating that their stress from time resource difficulties and colleague relation were quite high. 


\subsection{Predictors for Teaching Anxiety and Teacher Stress}

To reveal predictors for teaching anxiety and teacher stress, step-wise regression analyses were run on the measured variables, with demographic variables and TSI scales being independent variables for the dependent variable TAS and the demographic variables and TAS scales being independent variables for the dependent variable TSI. The results are summarized in Table 4. As reported in Table 4, 3 models were resulted with the change in $\mathrm{R}^{2}$ being all significant for TAS: .309 for model 1 (TSI), .068 for model 2 (TSI \& professional title), and .013 for model 3 (TSI, professional title \& age). Of the three variables included in model 3, TSI (overall teacher stress) $(\beta=.536, t=10.78)$ was the most powerful predictor, followed by professional title $(\beta=-.179, \mathrm{t}=-2.93)$ and age $(\beta=-.142, \mathrm{t}=-2.33)$ respectively, with effect sizes being small to medium $\left(\mathrm{f}^{2}=.011 \sim .03\right)$. Meanwhile, regression analyses yielded 4 models with the change in $\mathrm{R}^{2}$ being all significant for TSI: .309 for model 1 (TAS), .135 for model 2 (TAS \& TAS4), .014 for model 3 (TAS, TAS4 \&years of teaching), and .009 for model 4 (TAS, TAS4, age \& TAS6). Among the four variables included in Model 4 , TAS $(\beta=.386, t=6.65)$ was the most powerful predictor, followed by TAS4 $(\beta=.374, \mathrm{t}=7.596)$, years of teaching $(\beta=.133, \mathrm{t}=2.82)$, and TAS6 $(\beta=.123, \mathrm{t}=2.11)$, with a small to medium effect size $\left(\mathrm{f}^{2}=.006 \sim .03\right)$.

Table 4. Multiple Regression Coefficients and Significance of Predictors for TAS and TSI

\begin{tabular}{cccccc}
\hline & \multicolumn{5}{c}{ TAS } \\
\cline { 2 - 6 } & $\beta$ & $\mathrm{t}$ & $\mathrm{p}$ & VIF & Cohen's f $^{2}$ \\
TSI & .536 & 10.78 & .000 & 1.02 & .03 \\
Professional title & -.179 & -2.93 & .004 & 1.55 & .018 \\
Age & -.142 & -2.33 & .021 & 1.53 & .011 \\
\hline & $\beta$ & $\mathrm{T}$ & $\mathrm{p}$ & VIF & Cohen's f $^{2}$ \\
TAS & .386 & 6.65 & .000 & 1.59 & .03 \\
TAS4 & .374 & 7.596 & .000 & 1.14 & .015 \\
Years of teaching & .133 & 2.82 & .003 & 1.05 & .007 \\
TAS6 & .123 & 2.11 & .005 & 1.61 & .006 \\
\hline
\end{tabular}

Notes: $* *=\mathrm{p} \leq .01 ; *=\mathrm{p} \leq .05$

effect size of Cohen's $f^{2}:$ small $=f^{2} \leq .02 ;$ medium $=f^{2}=.15 ; \quad$ large $=f^{2} \geq .35$ (Cohen, 1988)

\section{Discussion}

Reliability analysis, together with factor analyses and correlation analyses, showed that both the TAS and TSI scales were highly reliable, as found in many prior studies (Ahmed \& Julius, 2015; Ameen et al., 2002; Cubukcu, 2013; Fimian, 1984, Fimian \& Blanton, 1987; Fimian \& Fastenau, 1990; Keavney \& Sinclair, 1978; Parsons, 1973). This study also showed that TAS and TSI scales were highly related to each other, as found in Desouky and Allam (2017) and Ahmed and Julius (2015).

\subsection{Teaching Anxiety and Teacher Stress Levels}

As shown in Table 3, the participants scored around 2.50 on TAS and three TAS subscales, similar to the finding in studies using the same or adapted scale (Ahmed \& Julius, 2015; Ameen et al., 2002; Cubukcu, 2013; Parsons, 1973; Olthuis et al., 2014; Robinson \& Clay, 2005). Alternatively, like their counterparts at different educational levels in different contexts, the participants in the present study were generally not worried about classroom teaching (TAS1). Even so, the mean score showed that at least one third of the participant actually worried about classroom teaching, further confirming that anxiety is a big issue for teachers (Morton et al., 1997; Peker \& Halat, 2009; Wang \& Wang, 2015). Unlike Parsons (1973) and other researchers who treated TAS as a uni-dimensional construct, the present study ran factor analysis on TAS and revealed six underlying components. Statistical analyses further showed that the participants were generally interested and confident in teaching and being good teachers (TAS2) and were not much concerned with the effects of their teaching (TAS5), which echoed the findings in Wang and Wang (2015). This might be attributed to traditional Chinese culture which highly values education and views teachers as authority figures. In addition, the study found that the participants were generally anxious about research (TAS3), allocating time for different work (TAS4) (like balancing time for research and teaching, administrative work, and too much unrelated to teaching and research), and promotion (TAS6). This was largely due to the administrative system and increasing demand for research in institutes of higher education in China. 
Meanwhile, this study revealed that the respondents were under (quite) high stress of workload, professional/recognition needs, time resource difficulties and colleague relation (see Table 3), similar to or even higher than their counterparts in other contexts (Boyle et al., 1995; Desouky \& Allam, 2017; Fimian \& Blanton, 1987; Kyriacou, 1989, 2001; Pithers \& Fogarty, 1995). This was surprising in that their self-reported teaching anxiety level was not remarkably high. Nevertheless, this was understandable that university teachers in the contemporary era had to engage themselves in much more work beyond teaching. For example, they had to worry about income since teachers in China had not been paid much yet living expenses simply soared up; they suffered from the stress of multiple workload of teaching, research and administration, especially after the new plan of constructing "Double World-Class Project" Universities was issued by the government; meanwhile, their good work might not be well recognized due to complicated reasons, one of which was the lack of an open and fair evaluation system.

\subsection{Relationships between TAS and TSI Scales and Demographic Variables}

As presented in Table 4, the present study found that half TAS scales were significantly correlated with the demographic variables, consistent with the findings in the current literature (Morton et al., 1997; Peker \& Halat, 2009; Wang \& Wang, 2015; Yazici \& Altun, 2013). It is understandable that older, more experienced teachers and/or those with higher professional titles tend to be less concerned about classroom teaching, promotion and research, as found in the present study, in that they have secured everything and/or are approaching retirement. Teachers with higher education levels tend to be less anxious about research because they know better how to undertake research. Also, as expected, male participants reported to be less concerned with research in the present research.

Strangely, TSI seemed not to be much related to the demographic variables. This might be due to two reasons: a) the $\mathrm{p}$ levels were set to be too low, and b) the sample size of specific groups might differ a lot. Even more surprisingly, participants with higher professional titles tended to be under more stress for professional/recognition needs. Since few studies have examined relations between TSI and demographic variables, more research is called for to confirm these findings. To date, James and Perry's (1978) research indicated that salary and age affected teachers' mental health. Desouky and Allam's (2017) cross sectional study of 568 Egyptian teachers found that occupational stress and anxiety scores were significantly related to age, gender, salary, school level, teaching experience, qualification and workload.

\subsection{Predictors for Teaching Anxiety and Teacher Stress}

As previously discussed, the respondents reported various sources for their anxiety such as the poor career structure, poor administration, the imperfect teacher evaluation system, inadequate salary, difficulties in personal development, heavy workload and teacher-student relationships, as found in the current literature (Coates \& Thoresen, 1976; Joseph, 1975; Merç, 2011; Wang \& Wang, 2015). Most of these sources overlapped with the stressors, as shown in the subcomponents of the TSI in the present study and those identified in Selye (1976) and Kyriacou (2001), further confirming the close relationship between teaching anxiety and teacher stress. Meanwhile, regression analyses revealed that TSI (overall teacher stress) was a powerful positive predictor and professional title and age were powerful negative predictors for the participants' teaching anxiety level (TAS). It also showed that TAS (overall teaching anxiety), TAS4 (time difficulty), years of teaching, and TAS6 (concerns about promotion) were powerful positive predictors for TSI (see Table 4), largely consistent with the results of correlational analyses reported in Table 3. These findings indicate that professional title and age greatly affect anxiety levels in university teachers of arts. They further suggest that teaching anxiety and teacher stress are not only highly related to each other but mutually predictive. The only exception was that years of teaching which positively predicted TSI was only insignificantly related to TSI. This might be due to the interaction of various variables working in the context, as correlational analyses revealed a significant negative correlation between professional titles and stress for professional/recognition needs (TSI4). Yet it was still hard to explain this, which requires further scrutiny.

As discussed, at least one third of the participants were anxious and the majority of them were under high stress due to various reasons. This meant that anxiety and stress were serious issues for university teachers of arts in China, which need to be carefully handled, since anxiety and stress could lead to serious consequences as reviewed in the literature (Joseph, 1975; Keavney \& Sinclair, 1978; Silvernail \& Costello, 1983). To do this, self-awareness is crucial (Joseph, 195), implying that teachers should be aware of their own anxiety levels, stressors for their anxiety, students' behavior and attitudes as well as their family members' and colleagues'.

Training, observation of classroom teaching, being well-prepared can all help to reduce teaching anxiety (Joseph, 1975; Koran, Jr. \& Koran, 1981; Peker, 2009; Williams, 1991). Williams's (1991) experimental study investigated the effects of a comprehensive teaching assistant (TA) training program on the teaching anxiety and effectiveness of 
27 TAs in a research university, who participated in a one-week workshop and a sixteen-week theory and pedagogy course. The results showed that declines in teaching anxiety for TAs in the experimental group were significantly greater and that the teaching effectiveness of the experimental group was rated significantly higher. Peker's (2009) study of the effects of expanded microteaching on 43 pre-service mathematics teachers' teaching anxiety showed that using the expanded microteaching in teaching practicum course reduced the teaching anxiety levels of the teacher participants. In addition, role playing could help lower pre-service and beginning teacher anxiety (Johns, 1992).

As discussed in Kyriacou (2001) and Zhang and Niu (2008), teachers can cope with stress by taking the following strategies: avoiding confrontations, trying to relax after work, keeping feelings under control, discussing problems and expressing feelings to others, being aware of one's own strengths and weaknesses, and keeping on personal development. It is important for individual teachers to be aware of the existence of stress and discover what strategies work best for them (Roger \& Hudson, 1995). Meanwhile, a positive, mutually supportive work atmosphere can help reduce teacher stress, while poor administration and poor management can create unnecessary sources of stress in teachers (Kryiacou, 2001). As elaborated in Kryiacou (2001) and Zhang and Niu (2008), it is necessary to create a healthy work environment in schools/universities, which can be done by establishing consensus on key values and standards, making decisions based on consultation, ensuring good communication between staff, offering positive feedback and praise to teachers, providing adequate resources and facilities to support teachers, and making easy-to-follow policies and procedures. In addition, counselling services can be made available to staff who are experiencing high levels of stress, like the telephone 'helpline' for teachers called 'teacherline' in UK (Kryiacou, 2001).Continuous self-development is always a good motivator and enhances one's confidence and competence in work (Zhang \& Niu, 2008). Ferguson, Frost and Hall's (2012) study of 274 in-service K1-12 teachers in Canada showed that efforts made to improve student behavior and employment conditions might lead to reduced stress among teachers and thus lower levels of depression and anxiety. It should be equally effective for university teachers.

\section{Conclusions, Implications and Suggestions for Future Research}

As Fimian discussed (1984), anxiety and stress are complex issues, which can be attributed to numerous factors. The present study revealed that university teachers of arts were under great stress, the sources of which could be attributed to heavy workload, a poor career structure, colleague relations, difficulties in planning time and resources, needs for recognition and so on. These sources were also reported to be sources of anxiety by the participants, though they were generally not so anxious about teaching. These findings further show that anxiety and stress in university teachers are highly related to and mutually predict each other, as found in the present study, which calls for efforts of individual teachers, schools and universities, and even local governments to help reduce anxiety and stress in university teachers (Zhang \& Niu, 2008).

This study revealed important findings about anxiety and stress in university teachers of arts in China, but limitations still existed. Firstly, only university teachers of arts were involved in the present though it meant for university teachers of all subjects. The reasons for the absence of university teachers of other disciplines might be interestingly intricate, which, together with their anxiety and stress levels, deserve research to reveal a fuller picture of anxiety and stress in in-service university teachers in China. Moreover, due to the big difference in sample sizes of specific participant groups (e.g., male and female participants, participants in different age groups, with different education levels, professional titles and years of teaching), no further statistical analyses were run to explore the differences between these specific samples. Future research may direct towards this to better identify features and differences in anxiety and stress in in-service university teachers with various backgrounds. In addition, as discussed in Buitink and Kemme (1986), teaching anxiety is momentary and situational and thus may change in intensity as time goes on and experiences accumulate. It is the same with teacher stress. Hence, it is worthwhile to explore the changes in teaching anxiety and teacher stress in cross-sectional studies.

\section{References}

Agustiana, V. (2014). Pre-service teachers' anxiety during teaching practicum. English Review: Journal of English Education, 2(2), 174-182. https://journal.uniku.ac.id/index.php/ERJEE/article/view/104/65

Ahmed, Z., \& Julius, S. H. (2015). The relationship between depression, anxiety and stress among women college students. Indian Journal of Health and Wellbeing, 6(12), 1232-1234.

Ameen, E.C., Guffey, D. M., \& Jackson, C. (2002). Evidence of teaching anxiety among accounting educators. Journal of Education for Business, 78(1), 16-22. https://doi.org/10.1080/08832320209599692

Betoret, F. D. (2006) Stressors, self-efficacy, coping resources, and burnout among secondary school teachers in 
Spain. Educational Psychology, 26(4), 519-539. https://doi.org/10.1080/01443410500342492

Boyle, G. J., Borg, M. G., Falzon, J. M., \&Baglioni Jr., A. J. (1995). A structural model of the dimensions of teacher $\begin{array}{lllll}\text { stress. British Journal of Educational } & \text { Psychology, } & 65(1), & \text { 49-67. }\end{array}$ https://doi.org/10.1111/j.2044-8279.1995.tb01130.x

Buitink, J., \& Kemme, S. (1986). Changes in student-teacher thinking. European Journal of Teacher Education, 9(1), 75-84. https://doi.org/10.1080/0261976860090109

Coates, T. J., \& Thoresen, C. E. (1976). Teacher anxiety: A review with recommendations. Review of Educational Research, 46(2), 159-184. https://doi.org/10.3102/00346543046002159

Cohen, J. (1988). Statistical power analysis for the behavioral sciences $\left(2^{\text {nd }}\right.$ ed.). New Jersey: Lawrence Erlbaum Associates.

Cubukcu, F. (2013). The significance of t academic emotions. Procedia-Social and Behavioral Sciences, 70, 649-653. https://doi.org/10.1016/j.sbspro.2013.01.105

Desouky, D., \& Allam, H. (2017). Occupational stress, anxiety and depression among Egyptian teachers. Journal of Epidemiology and Global Health, 7, 191-198. https://doi.org/10.1016/j.jegh.2017.06.002

Ferguson, K., Frost, L., \& Hall, D. (2012). Predicting teacher anxiety, depression, and job satisfaction. Journal of Teaching and Learning, 8(1), 27-42. https://doi.org/10.22329/jtl.v8i1.2896

Fimian, M. J. (1984). The development of an instrument to measure occupational stress in teachers: The Teacher Stress Inventory. Journal of Occupational Psychology, 57, 277-293. https://doi.org/10.1111/j.2044-8325.1984.tb00169.x

Fimian, M. J., \& Blanton, L. P. (1987). Stress, burnout, and role problems among teacher trainees and first-year teachers. Journal of Occupational Behavior, 8, 157-165. https://doi.org/10.1111/j.2044-8325.1984.tb00169.x

Fimian, M. J., \& Fastenau, P. S. (1990). The validity and reliability of the Teacher Stress Inventory: A re-analysis of aggregate data. Journal of Organizational Behavior, 11, 151-157. https://doi.org/10.1002/job.4030110206

Gardner, L. \& Leak, G. (1994). Characteristics and correlates of teaching anxiety among college psychology teachers. Teaching of Psychology, 21(1), 28-32. https://doi.org/10.1207/s15328023top2101_5

Hiebertt, B., \& Farber, I. (1985). Teacher Stress: A Literature Survey with a Few Surprises. Canadian Journal of Education, 9(1), 14-27. https://doi.org/10.2307/1494447

Hismanoglu, M. (2013). Foreign language anxiety of English language teacher candidates: A sample from Turkey. Procedia - Social and Behavioral Sciences, 93, 930-937. https://doi.org/10.1016/j.sbspro.2013.09.306

İpek, H. (2016). A qualitative study on foreign language teaching anxiety. Journal of Qualitative Research in Education, 4(3), 92-105. https://doi.org/10.14689/issn.2148-2624.1.4c3s5m

James, J., Perry, P. (1978). Assessing teacher stress: A beneficial task for the administrator. American Secondary Education, 8(4), 49-57. https://www.jstor.org/stable/41063244

Johns, K. M. (1992). Lowering beginning teacher anxiety about parent-teacher conferences through role-playing. The School Counselor, 40(2), 146-152. https://www.jstor.org/stable/23901154

Joseph, P. B. (1975). Value Conflict in Law-Focused Education: The Problem of Teacher Anxiety. Social Education, 1, 166-169. https://www.researchgate.net/publication/322927162

Keavney, G., \& Sinclair, K. E. (1978). Teacher Concerns and Teacher Anxiety: A Neglected Topic of Classroom Research. Review of Educational Research Spring, 48(2), 273-290. https://doi.org/10.3102/00346543048002273

Koran, Jr., J. J., \& Koran, M. L. (1981). The effects of teacher anxiety and modeling on the acquisition of a science teaching skill and concomitant student performance. Journal of Research in Science Teaching, 18(4), 361-370. https://doi.org/10.1002/tea.3660180411

Kyriacou, C. (2001). Teacher stress: Directions for future research. Educational Review, 53(1), 27-35. https://doi.org/10.1080/00131910120033628

Levine, G. (1996). Variability in anxiety for teaching mathematics among pre-service elementary school teachers enrolled in a mathematics course. Paper presented at the Annual Meeting of the American Educational Research Association in New York. (ERIC Document Reproduction Service No. ED398067). https://eric.ed.gov/?id=ED398067 
Machida, T. (2011). Teaching English for the first time: Anxiety among Japanese elementary school-teachers (Unpublished Ph.D dissertation). University of Illinois at Urbana-Champaign, USA. http://hdl.handle.net/2142/24324

Mandler, G., \& Sarason, S. B. (1952). A study of anxiety and learning. Journal of Abnormal and Social Psychology, 47, 166-173. https://doi.org/10.1037/h0062855

Merç, A. (2011). Sources of foreign language student teacher anxiety: A qualitative inquiry. Turkish Online Journal of Qualitative Inquiry, 2(4), 80-94. https://eric.ed.gov/?id=ED537806

Morton, L. L., Vesco, R., Williams, N. H., \& Awender, M. A. (1997). Student teacher anxieties related to class management, pedagogy, evaluation, and staff relations. British Journal of Educational Psychology, 67, 69-89. https://doi.org/10.1111/j.2044-8279.1997.tb01228.x

Olthuis, J. V., Watt, M. C., \& Stewart, S. H. (2014). Disorders Anxiety Sensitivity Index (ASI-3) subscales predict unique variance in anxiety and depressive symptoms. Journal of Anxiety Disorders, 28, 115-124. https://doi.org/10.1016/j.janxdis.2013.04.009

Parsons, J. S. (1973). Assessment of anxiety about teaching using the Teaching Anxiety Scale: Manual and research report. Paper presented at the Annual Meeting of American Educational Research Association in New Orleans, Louisiana. https://eric.ed.gov/?id=ED079330

Peker, M. (2009). The use of expanded microteaching for reducing preservice teachers' teaching anxiety about mathematics. Scientific Research and Essay, 4(9), 872-880. http://www.academicjournals.org/SRE

Peker, M., \& Halat, E. (2009). Teaching anxiety and the mathematical representations developed through webquest and spreadsheet activities. Journal of Applied Sciences, 9(7), 1301-1308. https://doi.org/10.3923/jas.2009.1301.1308

Pithers, R. T., \& Fogarty, G. J. (1995). Symposium on teacher stress: Occupational stress among vocational teachers. British Journal of Educational Psychology, 65, 3-14. https://doi.org/10.1111/j.2044-8279.1995.tb01127.x

Robinson, J. H., \& Clay, D. L. (2005). Potential school violence: Relationship between teacher anxiety and warning-sign identification. Psychology in the Schools, 42(6), 623-635. https://doi.org/10.1002/pits.20100

Roger, D., \& Hudson, C. (1995). The role of emotion control and emotional rumination in stress management training, International Journal of Stress Management, 2, 119-132. https://doi.org/10.1007/BF01740298

Seyle, H. (1976). The stress of life. New York: McGraw Hill.

Silvernail, D. L., \& Costello, M. H. (1983). The impact of student teaching and internship programs on preservice teachers' pupil control perspectives, anxiety levels, and teaching Concerns. Journal of Teacher Education, 34(4), 32-36. https://doi.org/10.1177/002248718303400409

Spielberger, C. D. (1966). Theory and research on anxiety. In C. D. Spielberger (ed.), Anxiety and behavior (pp. 3-20). New York: Academic Press. https://doi.org/10.1016/B978-1-4832-3131-0.50006-8

Spielberger, C. D. (1983). Manual for the State-Trait Anxiety Inventory (Form Y). Palo Alto, California: Consulting Psychologists Press. https://doi.org/10.1037/t06496-000

Wang, X., \& Wang, Y. (2015). Analysis on the state anxiety of college English teachers during transitional period of college English teaching reform and coping strategies. Foreign Language Learning Theory and Practice (FLLTP), 2, 31-38. http://www.cnki.com.cn/Article/CJFDTotal-GWJX201502006.htm

Westerback, M. E. (1982). Studies on attitude toward teaching science and anxiety about teaching science in pre-service elementary teachers. Journal of Research in Science Teaching, 19(7), 603-616. https://doi.org/10.1002/tea.3660190710

Williams, L. S. (1991). The effects of a comprehensive teaching assistant training program on teaching anxiety and effectiveness. Research in Higher Education, 32(5), 585-598. https://doi.org/10.1007/BF00992630

Yazici, H., \& Altun, F. (2013). Conditions of beginning teaching and anxiety perceived in teacher Candidates. Procedia-Social and Behavioral Sciences, 93, 974-977. https://doi.org/10.1016/j.sbspro.2013.09.313

Zhang, Y., \& Niu, X. (2008). A study of anxiety and depression in middle school teachers. Theory and Practice of Education, 6, 42-44. http://www.cnki.com.cn/Article/CJFDTotal-JYLL200817022.htm 